\title{
Sobre la evolución y el tránsito del Paleolítico medio al superior en el Pirineo occidental: un proyecto de hipótesis
}

\author{
Andoni Sáenz de Buruaga *
}

\begin{abstract}
RESUMEN ABSTRACT
Una buena parte de los yacimientos musterienses estratificados del País Vasco incluyen testimonios objetivos, sedimentológicos y faunísticos, de condiciones climáticas rigurosas en momentos avanzados del Paleolítico medio, próximos a 40000 B.P. Asociados a esos particulares depósitos se advierten una serie de indicios denunciantes de innovaciones y cambios en la composición de los conjuntos industriales y en la gestión del territorio; transformaciones plenamente orientadas hacia la dinámica propia del Paleolítico superior. Estos acontecimientos, climáticos y paletnológicos, pueden relacionarse y explicarse conjuntamente desde un punto de vista evolutivo a partir de un episodio crítico en el que confluyen simultáneamente varios de estos hechos. Se trata,

A significant part of stratified Mousterian sites in the Basque Country comprises objective, sedimentary and fauna testimonies of harsh climatic conditions in advanced stages of Middle Palaeolithic, which could be chronologically dated around 40000 B.P. There is some evidence associated to these particular deposits proving innovations and changes in the compositions of industrial assembiages and territory management. These transformations are completely oriented towards the typical dynamics of Upper Palaeolithic. All these climatic and paletnologic events can be linked and explained together from an evolutionary point of view starting from a critical episode where some of these facts occurred simultaneously. This is, undoubtedly, the point of
\end{abstract}

* Departamento de Geografía, Prehistoria y Arqueología. Facultad de Filología, Geografía e Historia. Universidad del País Vasco-Euskal Herriko Unibertsitatea. C/ Francisco Tomás y Valiente, s/n. 01006 Vitoria-Gasteiz. 
verdaderamente, del comienzo del proceso de cambio irreversible del

Paleolítico medio al superior.

PALABRAS CLAVE: Paleolítico medio, Musteriense, Crisis evolutiva, País Vasco. departure for a process of irreversible transition from Middle to Upper

Palaeolithic.

KEY WORDS:

Middle Palaeolithic, Mousterian,

Evolutionary crisis, Basque Country.

La incidencia del clima en el comportamiento y evolución de las industrias y de otras variables humanas es un argumento clásico varias veces utilizado para explicar la evolución de los complejos sociales prehistóricos.

En el marco geográfico del País Vasco, por tanto en un espacio en relación directa con el Pirineo (hacia el E) y el Cantábrico (hacia el W), comienzan a percibirse algunos indicios de importantes transformaciones paletnológicas que pueden plantearse en relación con un episodio climático riguroso en torno al 40000 B.P.

Varios datos advertidos en la composición tipológica y tecnológica de los ajuares industriales, y otros hechos, más puntuales, asociados a la captación de materias primas y a la misma organización del registro estratigráfico -exponentes ambos de una determinada concepción territorial-, posibilitan considerar seriamente la existencia de significativas modificaciones de orden paletnológico en esas fases avanzadas del Paleolítico medio. Transformaciones que parecen orientarse directamente hacia la dinámica industrial y espacial que se generalizará en este contexto durante la mayor parte del Paleolítico superior.

En efecto, desde nuestro razonamiento, la gestación de los complejos industriales iniciales del Paleolítico superior estaría en relación directa con la formación y desarrollo evolutivo de una serie de complejos industriales protoleptolíticos que a lo largo de esos episodios avanzados y terminales del Paleolítico medio entrarán en una definitiva fase de aceleración evolutiva cualitativa. Asistiéndose a una, a veces, pausada, en casos, más sensible, leptolitización de los complejos musterienses. Además, paralelamente, algunos hechos puntuales vincularían directamente las estrategias de ocupación y explotación del territorio en esos momentos con las pautas de gestión espacial, mejor conocidas, entre el Auriñaciense y el Magdaleniense en buena parte de ese mismo marco físico.

En otras palabras, trabajamos con la hipótesis de que el paso del Paleolítico medio al superior está en relación con un proceso de acelera- 
ción evolutiva de ciertos caracteres paletnológicos impulsado por un incremento de la rigurosidad climática en torno a 40000 B.P.

A pesar de que, si bien, varios de los sucesos revelados se apoyan en sólidos testimonios materiales, es cierto que algún otro se encuentra, provisionalmente, más limitado en cuanto a las observaciones que lo sustentan. Es por ello que, a pesar de constituir en su globalidad un conjunto de hechos concatenados, y a la espera de venideras comprobaciones $y$ de los nuevos datos a aportar por las varias excavaciones actualmente (2003) en curso (Abauntz, Axlor, Isturitz, Lezetxiki, Venta Laperra o Zerratu son ejemplo de ello), planteamos nuestra propuesta con una razonable cautela. En la dinámica de investigación la prudencia debe marchar en la misma vía que el rigor científico y la creatividad a la hora de plantear una hipótesis.

\section{MARCO FÍSICO Y FUENTES DE CONOCIMIENTO}

A pesar de que varias de las ideas aquí contenidas pudieran refrendarse en contextos geográficos más amplios, conviene explicitar que hemos preferido comedir su desarrollo al marco territorial que más intensamente hemos reconocido, el del País Vasco.

En este contexto se tiene constancia de un notable elenco de ejemplos ilustrativos del Paleolítico medio.

Los yacimientos más notables se corresponden con varias cuevas y abrigos como los de Arrillor en Álava, Amalda, Lezetxiki y Zerratu en Gipuzkoa, Abauntz y Coscobilo en Navarra, Olha y Olha 2 en Laburdi, Isturitz en Bajanavarra y Gatzarria y Harregi en Zuberoa. A ellos habría que añadir las importantes estaciones al aire libre de Murba en Álava, Kurtzia en Bizkaia, Urbasa en Navarra y Basté en Laburdi. Además, varias referencias aisladas se han recuperado sobre un considerable número de sitios de superficie: de terrazas fluviales del Ebro y del Adour, del litoral costero de Gipuzkoa y de Laburdi, así como de algunas formaciones montañosas interiores de Álava, Navarra y Bajanavarra.

De cara al conocimiento específico de cada una de las situaciones arqueológicas se cuenta con un valioso número de informaciones publicadas.

Como más relevantes deben citarse, en primer término, las memorias de excavación de yacimientos, como Axlor (por J. M. de Barandiarán en 1980), Basté (por Cl. Chauchat en 1968), Isturitz (por R. y M. de SaintPérier en 1952), Kurtzia (por J. M. de Barandiarán, A. Aguirre y M. Grande 
en 1960), Lezetxiki (por J. M. de Barandiarán en 1978), Murba (por A. Baldeón en 1988), Olha (por E. Passemard en 1924) y Venta Laperra (por J. M. de Barandiarán en 1958), y el estudio pluridisciplinar de alguno de ellos como el de Amalda (por J. Altuna, A. Baldeón y K. Mariezkurrena en 1980). De obligada consulta es la monografía de síntesis sobre el Paleolítico en Euskal Herria de I. Barandiarán de 1988.

Son asimismo de elevado interés:

- los análisis tipológicos de varios conjuntos industriales musterienses, como los de Axlor (por A. Baldeón en 1999), Coscobilo (por M.A. Beguiristain en 1974), Gatzarria (por G. Laplace y A. Sáenz de Buruaga en 2003), Harregi (por A. Sáenz de Buruaga y D. Ebrard), s.f., Lezetxiki (por A. Baldeón en 1993), Mugarduia en Urbasa (por I. Barandiarán y L. Montes en 1992), y Olha 2 (por G. Laplace y A. Sáenz de Buruaga en 2000);

- los trabajos de caracterización sedimentológica de Arrillor (por M. Hoyos, A. Sáenz de Buruaga y A. Ormazabal en 1999), Gatzarria (por F. Lévêque y J-Cl. Miskovsky en 1996) y Lezetxiki (por P. Kornprobst y P. Rat en 1967); $y$,

- los estudios globales de series faunísticas como los de J. Altuna de 1972 y F. Lavaud de 1980.

Además, avances de las últimas campañas de excavación pueden seguirse anualmente en la serie «Arkeoikuska» como para Arrillor (por A. Sáenz de Buruaga, de 1989 a 1994 y 1997), Axlor (por J. González Urquijo y J. J. Ibáñez, a partir de 1999), Kurtzia (por M. Muñoz Salvatierra de 1984 a 1989), Lezetxiki (por A. Arrizabalaga, a partir de 1996), Venta Laperra (por R. Ruiz ldarraga, a partir de 2001) y Zerratu (por A. Sáenz de Buruaga y J. A. Mujika, a partir de 2002).

Nuestro propósito es hacer una serie de reflexiones sobre la constatación de unos hechos en este marco físico del Pirineo occidental e inmediato contexto cantábrico, algunos de ellos bien refrendados bibliográficamente, otros derivados del estudio personal de los complejos industriales y de la excavación de algunos yacimientos, y aprovechar la circunstancia para plantear una hipótesis explicativa de los mismos.

Una hipótesis -reiteramos- que relaciona en los momentos avanzados del Paleolítico medio la incidencia de un fenómeno climático objetivo (el frío) con algunos indicios de alteraciones paletnológicas orientadas en la dinámica industrial y territorial del Paleolítico superior. $Y$ entra las que habría que enunciar: 
- la formación y desarrollo evolutivo de una serie de complejos industriales protoleptolíticos o conjuntos maeriales musteroides enriquecidos de formas y técnicas propias del Paleolítico superior; y,

- ciertas innovaciones en la gestión del espacio formulables a partir de algunos cambios en la captación de las materias primas y en la organización estratigráfica de los yacimientos.

\section{TESTIMONIOS OBJETIVOS DE ALTERACIONES CLIMÁTICAS RI- GUROSAS EN TORNO A 40000 BP}

La observación detallada de los depósitos musterienses pirenaicos y cantábricos controlados en torno al País Vasco revela la existencia de condiciones climáticas rigurosas en los tramos superiores y terminales de una buena parte de los yacimientos investigados.

Así, parece desprenderse de los estratigráficamente más representativos y, además, extensivamente excavados, como Arrillor (Álava), Axlor (Bizkaia), Gatzarria (Zuberoa), Harregi (Zuberoa), Isturitz (Bajanavarra), Lezetxiki (Gipuzkoa), Olha y Olha 2 (Laburdi) y Zerratu (Gipuzkoa). Es decir, en la mayoría del catálogo de yacimientos musterienses estratificados del País Vasco.

De hecho, quedarían sólo al margen de esta consideración un pequeño número de cuevas y algunas estaciones al aire libre.

Entre las primeras, habría que citar las de Abauntz (Navarra), en proceso de excavación, Amalda (Gipuzkoa), con un solo nivel musteriense (el VII) para el que se propuso una formación muy antigua (Würm I), Coscobilo (Navarra), cuyo depósito se encontraba destruido, a consecuencia de una explotación de cantera, con antelación a su conocimiento arqueológico, y Venta Laperra (Bizkaia), de donde se conoce un antiguo sondeo y actualmente se excava.

Entre las segundas, frecuentemente acompañadas de serias alteraciones postdeposicionales, deberían enumerarse los casos de Basté (Laburdi), con un nivel arqueológico musteriense (el 4), comprendido en el estrato $E$, que se ha hecho corresponder con los inicios del Würm II, y los de los talleres de Kurtzia (Bizkaia), en la misma línea de costa (datado indirectamente por la formación dunar de Atxiribil/Larrabasterra en $41400 \pm$ 2500 bp) y en conexión con los depósitos de sílex del flysch calcáreo del Cretácico superior y de los nódulos encajados en olistostromos del flysch detrítico de similar episodio mesozoico, de Murba (Álava), junto a un paleocauce del río Ayuda y donde se aprovechó la presencia de importantes 
aportes de sílex del Mioceno continental de la depresión de Treviño, y de Urbasa (Navarra), con varios sitios de superficie (como Andasarri, Aziarri, Gorlasaro, Mugarduia Norte,...) relacionados con la explotación de los importantes afloramientos de sílex paleoceno marino locales especialmente bien presentes en los escarpes rocosos que delimitan el perímetro septentrional del altiplano.

En conexión con nuestra afirmación de partida, en efecto, en varias ocasiones se han citados claros testimonios sedimentológicos y faunísticos de recrudecimiento climático en los registros estratigráficos temporalmente más avanzados del Paleolítico medio en el País Vasco. Depósitos que preceden inmediatamente a las primeras manifestaciones industriales auriñacoides y/o que culminan la sucesión de secuencias musteroides.

Así, por una parte, se han señalado formaciones de origen crioclástico (a base de deposiciones de bloques, clastos, de formaciones de cailloutis,...) en el conjunto estratigráfico superior de Arrillor (niveles, de arriba a abajo, Lam, Lmc -datado en $37100 \pm 1000$ bp-, Smb y Smc -que sucede, tras un neto y brusco contacto erosivo, al importante y denso depósito estratigráfico medio, de génesis fluvial, climáticamente más templado y húmedo, y del que se poseen fechaciones precisas para su tramo meso-inferior (nivel Amk) de $45700 \pm 1200$ bp y $45400 \pm 1800$ bp y para su parte meso-superior (nivel Smkl) de $43100 \pm 1700 \mathrm{bp}$-), en el nivel IV de Lezetxiki (al que se ha vinculado climáticamente con el Würm II y por encima del que se situarán directamente los complejos industriales ya propios del Paleolítico superior inicial), en el conjunto estratigráfico medio de Olha 2 (integrado por las capas, de arriba a abajo, Ask, Askf1, Ak y Askf2, todas ellas constitutivas del complejo industrial As, y cuya formación se ha relacionado con una fase fría del pleniglaciar inferior alpino meso-würmiense o Würm II) y en el conjunto estratigráfico Eab de Zerratu (en la parte meso-superior de la secuencia estratigráfica y constituido por los niveles, de arriba a abajo, Alab -éste incluyendo dos horizontes arqueológicos bien diferenciados en su posición: uno en la parte superior o Alabs, definiendo el techo de los depósitos arqueológicos del yacimiento, y otro en la parte inferior o Alabi-y Alabc).

Simultáneamente, por otra parte, en algunos de esos mismos depósitos crioclásticos y en otros registros musterienses somitales, cuyos testimonios en restos animales han sido suficientemente estudiados y descritos, se advierte la presencia en estas latitudes de claros indicadores faunísticos fríos. Bien, por medio de restos de reno (como en los niveles 4 de Axlor - cuya transición con el suprayacente 3 se ha datado en 
$41900 \mathrm{bp}$ - SIV o M de Isturitz —al que suceden los primeros indicios industriales propios del Paleolítico superior-, en el ya citado IV de Lezetxiki y en el conjunto medio $\mathrm{Fm}$ de Olha), bien, a pesar de ser algo menos sensible a las variaciones climáticas que el precedente, de rinoceronte lanudo (como en el nivel Lmc de Arrillor), o, incluso, junto a ambas especies, de mamut (como en el nivel Cj de Gatzarria - cuya formación se ha relacionado con una fase fría avanzada del pleniglaciar inferior alpino meso-würmiense o Würm Ilb y que aparece sucedido inmediatamente por el complejo industrial castelperroniense del nivel Cjn 3-, en el depósito superior Fs de Olha y en el conjunto arqueológico III/IV de Harregi).

Así pues, no parece aleatorio que las secuencias estratigráficas superiores de un buen número de yacimientos musterienses del País Vasco aporten señales inequívocas de una clara sintomatología climática fría. Son numerosas las observaciones sedimentológicas y faunísticas que corroboran la severa circunstancia. Se trata, hasta el momento, de los testimonios objetivos más sensibles de los rigores climáticos en este contexto geográfico desde el comienzo de la glaciación de Würm.

La posición estratigráfica de estos particulares depósitos, su coincidencia frecuentemente con los tramos somitales y terminales de las secuencias musterienses, a veces inmediatamente antecediendo a las primeras manifestaciones industriales del Paleolítico superior, unido, complementariamente, al —aunque todavía bastante deficiente- espectro de dataciones absolutas, directas e indirectas, disponibles, tenderían a situar estos acontecimientos climáticos en torno a $40000 \mathrm{bp}$.

\section{INDICIOS DE MODIFICACIONES PALETNOLÓGICAS RELATIVA- MENTE COETÁNEAS}

Una serie de interesantes alteraciones paletnológicas parecen sucederse simultánea y derivadamente de esos momentos climáticos rigurosos, a juzgar por ciertos indicios reveladores controlados en algunos de esos mismos contextos estratigráficos.

Bajo esta perspectiva deberán de considerarse como tales algunas notables innovaciones tipológicas y tecnológicas en la composición de los conjuntos industriales, acompañadas, probablemente, en algún caso, de cambios significativos en la concepción del espacio y, acaso, consecuencia de ello, de ciertas modificaciones en la ocupación y función de los asentamientos. 


\section{a) Innovaciones tecno-tipológicas en la industria lítica}

Un repertorio industrial evolucionado en el que, junto a elementos de substrato musteriense (raederas, denticulados, puntas), se advierte un cierto desarrollo de tipos de marcada orientación leptolítica y, eventualmente, de formas vinculadas tecnológicamente al debitado laminar, caracteriza la composición de algunos de los ajuares industriales propios de esos episodios fríos, como en Arrillor (niveles Lam y Lmc), Gatzarria (nivel $\mathrm{Cj}$ ), Olha 2 (niveles Ask, Askf1 y Askf2, del complejo industrial meso-superior As) y muy probablemente en Isturitz (nivel SIV) y Lezetxiki (nivel IV).

El carácter protoleptolítico de estos nuevos conjuntos viene determinado por la presencia, a veces simultánea, de una serie de rasgos tecno-tipológicos particulares. Así, deben, al menos, señalarse como indicios probables de esta sintomatología:

1. El desarrollo de algunos tipos habitualmente bien asociados a los conjuntos industriales evolucionados del Leptolítico, como, especialmente, raspadores, truncaduras, becs y buriles, lo que directamente conlleva una mayor diferenciación tipológica de estas series.

2. La presencia sensible de objetos de tendencia laminar y de verdaderas láminas, sean éstas derivadas de la técnica levallois o a partir de núcleos de láminas polarizados.

3. El desarrollo profuso y variado de algunas formas específicas, verdaderos paradigmas de la tendencia al alargamiento y estrechez de los tipos: nos referimos, especialmente, a las puntas de retoque simple que, en ciertos tipos unilaterales, se encuentran muy próximas ya de las puntas de dorso tipo Châtelperron o La Gravette. Digamos, en este sentido, que de estas puntas simples a las puntas de dorso hay un pequeño paso, que su tránsito es relativamente sencillo. Pudiera afirmarse que, efectivamente, el tipo parece hallarse ya concebido en las puntas simples y que la diferencia con los ejemplares de dorso vendrá dada por la disposición sobre ellas de un retoque algo más abrupto.

4. Más complementariamente, pudiera añadirse a esta relación la búsqueda, en productos de debitado no laminares, de formas alargadas y estrechas, como además de las puntas referidas, parece advertirse en varios tipos de raederas laterales.

La innovación tecno-tipológica supondrá, pues, la aparición de nuevos temas, el desarrollo de variaciones en los ya existentes y la práctica de diferentes ensayos sobre los habituales. 
Se trata, en consecuencia, de conjuntos industriales musteroides sensiblemente enriquecidos de formas protoleptolíticas en grados diversos, pues convendrá observar que en el seno de todo proceso de innovación se sucederán diferenciados movimientos y ritmos innovadores.

En este sentido, quizás, no resultaría ilusorio plantear teóricamente una vinculación con estos complejos industriales protoleptolíticos de algunos de los muy evolucionados conjuntos industriales localizados en el Cantábrico central, en torno a la cueva del Castillo, donde se ha asegurado la alta presencia de piezas auriñacoides en cronologías próximas a 40000 B.P.

\section{b) Cambios en la concepción del territorio de explotación y en la fun- ción táctica de los asentamientos}

El nivel Lmc de Arrillor procura, por el momento, el rastro más expresivo de nuevos movimientos en la dinámica paletnológica. Veamos la sucesión de hechos.

En ese depósito crioclástico, de en torno a 37000 B.P., junto a un repertorio industrial musteroide enriquecido de formas protoleptolíticas, se ha controlado, por vez primera en la amplia secuencia arqueológica del yacimiento, una importante variabilidad geográfica en relación al origen de las rocas silíceas utilizadas; diversidad que, en sus términos precisos, lleva a sugerir cambios significativos en las estrategias de captación de las materias primas.

En efecto, pudiera afirmarse que la más antigua tradición de aprovisionamiento de recursos líticos locales es sucedida en estos momentos por un repertorio material geográficamente más variado y extenso, de amplitud más regional, que hace entrever una novedosa concepción táctica del espacio, del territorio de explotación.

En este sentido, además de los aportes de sílex local se tiene constancia de la presencia en Arrillor de materiales silíceos alóctonos, originarios, al menos, de tres áreas geográficas del País Vasco bien diferenciadas:

-- de los depósitos terciarios continentales de Treviño, a unos $35 \mathrm{~km}$ al S;

- de los bancos terciarios marinos de Urbasa, a unos $55 \mathrm{~km}$ al E.SE; $y$,

- de los afloramientos del flysch cretácico del litoral vizcaíno, a unos $50 / 60 \mathrm{~km}$ al N.NE.

$Y$ es que, precisamente, se trata de los tres focos de aprovisionamiento lítico básico que caracterizarán, en términos generales, los complejos in- 
dustriales de los cazadores-recolectores del Paleolítico superior en buena parte del Cantábrico oriental.

En consecuencia, a juzgar por los indicios aquí referidos, cabría prudentemente aseverar que el espacio parece dejar de equipararse con un marco territorial restringido, más bien de definición local, para adquirir una mayor incidencia territorial extensiva, de ámbito más regional. Un comportamiento estratégico innovador y precursor de la dinámica de explotación territorial característica del Paleolítico superior, especialmente de la que parece mejor percibirse en sus episodios climáticos fríos y de la que el caso de Arrillor se ofrece muy cercano.

Muy probablemente, la nueva conducta territorial conllevaría ciertas modificaciones en la función táctica de algunos emplazamientos y en los ritmos de sus ocupaciones.

Así, quizás bien pudiera tener relación con una modificación en la función desempeñada por algunos yacimentos el hecho que varios de ellos dejen, aparentemente, en estos momentos de ser ocupados definitivamente (como Olha/Olha 2 y Zerratu, ambos con conjuntos industriales exclusivamente musteroides) o que sólo vuelvan a serlo, tras una significativa ruptura en la continuidad de la ocupación, en fases más avanzadas del Paleolítico superior (como Harregi a partir del Gravetiense evolucionado, Abauntz del Solutrense o Arrillor en el Magdaleniense pleno).

La intensidad de las ocupaciones parece, pues, remitir en algunas estaciones. Y es que cabría suponer que, efectivamente, la amplificación del espacio conllevaría una densificación de la red de yacimientos con la inclusión de nuevos emplazamientos sobre el territorio, como pudiera tratarse de los casos, p.e., de las cuevas de Antoliñako koba (Bizkaia), Labeko koba (Gipuzkoa) o Santimamiñe (Bizkaia), todas ellas con depósitos basales auriñacoides, que se incorporarán ahora a los casos, más perseverantes, de Gatzarria, Isturitz o Lezetxiki.

Quizás este incremento en la extensión del territorio y en la densidad de los yacimientos bien pudiera tener una relación directa con ciertas alteraciones que se advierten en la misma organización vertical del registro estratigráfico. Donde las amplias y dilatadas sucesiones de niveles musterienses controladas en Arrillor (con 21 niveles y horizontes de ocupación sobre $5 \mathrm{~m}$ de depósito sedimentario), Axlor (con, al menos, 8 niveles sobre $4,5 \mathrm{~m}$ ), Lezetxiki (con, al menos, 4 niveles sobre otros tantos, $4 \mathrm{~m}$ ), Olha 2 (con 11 niveles y horizontes sobre casi $4 \mathrm{~m}$ ) o Zerratu (con, al menos, 9 niveles y horizontes sobre casi $7 \mathrm{~m}$ ), se verán sustituidas por secuencias menos densas y profusas de niveles y horizontes arqueológicos. Definitivamente se tiene la sensación que el ante- 
rior diseño estratigráfico vertical esta siendo relegado por otro de desarrollo más horizontal.

\section{EL PASO DEL PALEOLÍTICO MEDIO AL SUPERIOR DESDE LA PERSPECTIVA DE UNA CRISIS EVOLUTIVA}

Si finalmente se conviniera en aceptar estos hechos concernientes a las alteraciones industriales y a la gestión del territorio como síntomas válidos de transformaciones paletnológicas más generales, parecería lógico, asimismo, vincular su desarrollo con el coetáneo proceso de evolución climática rigurosa suficientemente testimoniado.

En este sentido, cabría identificar esa confluencia relativamente simultánea de varios sucesos relevantes, climáticos y paletnológicos, con un episodio de crisis evolutiva que, por los datos de que disponemos, circundaría los 40000 B.P.

Así, valorando como consecuencias de la crisis las transformaciones paletnológicas referidas, debería contemplarse como causa directa más probable la significativa incidencia climática.

Una implicación del clima, y del propio significado del concepto de crisis, entendidos como un impulso de aceleración evolutiva con significación cualitativa y transcendencia temporal en el seno de un proceso general de evolución, en el que ya se advierten movimientos aislados previos en esa misma dirección. Ello bien parece corroborarlo el nivel Amk de Arrillor, generado en una fase climática templada húmeda, en torno a 45000 B.P., con un conjunto industrial polimórfico en el que junto al substrato musteriense hay un desarrollo de protoformas leptolíticas, a base de raspadores, truncaduras, buriles e, incluso, alguna punta de dorso. A pesar de su posición en el tramo meso-inferior de la secuencia musteriense, se trata de uno de los conjuntos industriales más evolucionados tipológicamente. Sin embargo, ese gesto impulsivo quedará diluido en el seno de un movimiento general de evolución más estacionario al no adquirir significación cualitativa temporal. Igualmente, debiera citarse entre estos ensayos previos, el caso del complejo industrial Sm de Olha 2, que define la base de los depósitos arqueológicos del remarcable abrigo musteriense, formado en un episodio climático templado húmedo, probablemente de una fase antigua del Würm II, y cuyo ajuar industrial se muestra, casi con seguridad, como el más laminar de toda la amplia secuencia arqueológica.

Por consiguiente, la diversidad tipológica asociada al enriquecimiento en formas protoleptolíticas y al desarrollo de la laminaridad no deben en- 
tenderse, solamente, como una respuesta adaptativa ante un desafío climático, sino que, ya presentes, si bien de forma discontinua, en ensayos temporalmente previos y al parecer climáticamente diferentes, encontrarán en la adversa climatología rigurosa su más óptimo medio de propagación, experimentando un notable dinamismo en su ritmo de evolución. Acaso tuviera que ver en este impulso en la transmisión de los nuevos caracteres un mayor contacto entre los grupos humanos a causa de una alta restricción en el volumen de productividad animal y vegetal impuesta por las severas condiciones climáticas. Pues, por lo general, se conviene en que es en los episodios de crisis ambiental cuando parecen generarse los cambios más sensibles en las pautas de selección de productos.

La consecución de un nivel de complejidad estructural, como el que manifiestan los complejos industriales protoleptolíticos, pudo probablemente conllevar, con antelación, la aparición gradual y puntual de algún cierto tipo de propiedades nuevas en el devenir evolutivo. Esto es algo que razonablemente hay que contemplar. Sin embargo, la emergencia definitiva de esos gestos innovadores no comenzará a adquirir significación cualitativa hasta el momento en que se alcance el umbral crítico. En nuestro caso, este episodio de crisis lo hacemos corresponder con el severo recrudecimiento climático de en torno a 40000 B.P., conformándose a partir de este momento una fase de equilibrio inestable orientada progresivamente hacia la dinámica general del Paleolítico superior y que constituye verdaderamente el prolegómeno del proceso de cambio del Paleoítico medio al superior. El papel del clima en este proceso responderá, por tanto, al de agente favorecedor e impulsor de las innovaciones morfo-técnicas.

Por ello, la comprensión de este proceso desde un prisma evolutivo implicará contemplar, junto a factores externos en el proceso de aceleración crítica, la existencia de ciertas tendencias ortogenéticas de carácter más interno y particular.

La asunción, así, de esta perspectiva pudiera posibilitar considerar diferentes dinámicas de tránsito entre los complejos evolucionados del Musteriense y las primeras industrias del Auriñaciense. Ello significaría reconocer divergencias en el proceso de evolución del Musteriense cara a la constitución de los complejos iniciales del Paleolítico superior. Lo que conduciría a plantear, complementariamente, otras alternativas a la sucesión Castelperroniense/Protoauriñaciense. Pues complejos tecnológica y tipológicamente tan evolucionados como Olha 2 recuerdan muy seriamente los propios del Auriñaciense, lo que pudiera avalar, en ciertos casos, la existencia de vías de paso más directo. 
De la misma manera, debería valorarse con similares argumentos el proceso de prolongación de los complejos industriales musterienses en el tiempo, en forma de un Tardimusteriense, cada vez más presente en amplias zonas de la Península Ibérica.

Para concluir con esta serie de deseos y propósitos, incluso, cabría cuestionarse si, acaso, la a veces aludida «regresión demográfica» neandertaliana, de en torno a 40000 B.P., en contextos meridionales de la Península Ibérica, no tendría, efectivamente, más que ver con cambios táctico-funcionales en la gestión del espacio y de la consecuente ordenación de los yacimientos que con una debilitación cuantitativa del efectivo de los grupos humanos neandertales.

Con todo, lógicamente, el razonamiento aquí desarrollado ayudará mejor a entender las formas de cambio del Paleolítico medio al superior desde la evolución que desde la substitución. Y ello, reiteramos, conllevará aceptar en la dinámica particular de los complejos industriales, junto a procesos de discontinuidad, otras posibilidades de tránsito derivadas de episodios polimórficos inmediatos.

\section{BIBLIOGRAFÍA}

Altuna, J. 1972. Fauna de mamíferos de los yacimientos prehistóricos de Guipúzcoa. Munibe, 24. San Sebastián

Altuna, J., Baldeón, A. y Marieżurrena, K. 1988. La cueva de Amalda (Zestoa, País Vasco). Ocupaciones Paleolíticas y Postpaleolíticas. Eusko lkaskuntza. Fundación José Miguel de Barandiarán. Colección Barandiarán, 4. San Sebastián.

BALDEÓN, A. 1988. «El yacimiento de Murba». Estudios de Arqueología Alavesa, 16: 7-160.

Baldéón, A. 1993. «El yacimiento de Lezetxiki (Guipúzcoa, País Vasco). Los niveles musterienses". Munibe, 45: 3-97.

Baldé́n, A. 1999. «El abrigo de Axlor (Bizkaia, País Vasco). Las industrias líticas de sus niveles Musterienses». Munibe, 51: 9-121.

Barandiarán, I. 1988. Historia General de Euskalerria. Prehistoria: Paleolítico. Enciclopedia General llustrada del País Vasco. Ed. Auñamendi, San Sebastián.

Barandiarán, I. y Montes, L. 1992. "Ocupaciones del Paleolítico en Urbasa (Navarra). El sitio de Mugarduia Norte». Trabajos de Arqueología de Navarra, 10:21-67.

Barandiarán, J. M. 1958. «Excavaciones en Carranza: Bortal, Venta de Laperra, Polvorín». Vizcaya, 10: 49-52.

BarandiarÁN, J. M. 1978. «Exploración de la cueva de Lezetxiki en Mondragón (Memorias de los trabajos de 1956, 1957, 1959 y 1960 y 1961 a 1968)». Obras Completas, XIII: 423-480, XIV: 7-131 y 327-333. Ed. La Gran Enciclopedia Vasca, Bilbao.

BARANDIARÁN, J. M. 1980. «Excavaciones en Axlor (Campañas de 1967 a 1974)». Obras Completas, XVII:127-384. Ed. La Gran Enciclopedia Vasca, Bilbao.

Barandiarán, J. M., Aguirre, A. y Grande, M. 1960. La estación prehistórica de Kurtzia: Barrica, Sopelana. Servicio de Investigación Arqueológica de la Excma. Diputación Provincial de Vizcaya, Bilbao.

BeguiristalN, M. A. 1974, «La colección Barandiarán de Coscobilo, de Olazagutía. Contribución al estudio de la industria lítica del yacimiento». Príncipe de Viana, 136-137: 345-401.

CHAUCHAT, Cl. 1968. Les industries préhistoriques de la région de Bayonne, du Périgordien ancien à l'Asturien. Thèse de $3 e$. Cycle. Université de Bordeaux. 
Hoyos, M., SÁenz de Buruaga, A. y Ormazabal, A. 1999. "Cronoestratigrafía y paleoclimatología de los depósitos prehistóricos de la cueva de Arrillor (Araba, País Vasco)". Munibe, 51: 137-151.

Kornprobst, P. y Rat, P. 1967. Premiers résultats d'un étude géologique et paléoclimatique du remplissage paléolithique moyen et supérieur de Lezetxiki (Mondragón, Guipúzcoa). Munibe, 19: 247-260.

Laplace, G. y SÁEnz de Buruaga, A. 2000. «Application de la Typologie analytique et structurale à l'étude de l'outillage moustéroïde de l'Abri Olha 2 à Cambo (Kanbo) en Pays Basque". Paléo, 12: 261-324.

LAPLACE, G. y SÁENZ DE BURUAGA, A. 2003. «Typologie analytique et structurale des complexes du Moustérien de la Grotte Gatzarria (Ossas-Suhare, Pays Basque) et de leurs relations avec ceux de l'Abri Olha 2". Pyrenae, 33-34, 2002-2003, (en prensa).

Lavaud, F. 1980. Les faunes paléolithiques du Würm II et III dans de sud-ouest et le centreouest de la France. Thèse de 3e. Cycle. Université de Poitiers.

LÉVÊQUE, F. y MISKOVSKY, J-Cl. 1996. «Le pasaje du Paléolithique moyen au supérieur dans la Grotte Gatzarria de Suhare: dones sédimentologiques et comparaisons". Congrès National des sociétés historiques et scientifiques, 118e, Pau: 41-60.

PAssemard, E. 1924. Les stations Paléolithiques du Pays Basque et leurs relations avec les Terrasses d'alluvions. Bayonne.

SÁenz de Buruaga, A. y Ebrard, D. (s.f.). "El Musteriense de la cueva de Harregi (Haréguy): Harregiko karbea, en Aussurucq, Pays de Soule (Excavaciones: Pierre Boucher, 19541960)". Veleia, (en prensa).

SAINT-PÉRIER, R. y S. 1952. La Grotte d'Isturitz. III. Les Solutréens, les Aurignaciens et les Moustériens. Archives de l'Institut de Paléontologie Humaine, 25. Paris. 